\title{
Cistos peribiliares em leão-africano (Panthera leo) ${ }^{1}$
}

\author{
Ricardo B. Lucena ${ }^{2}$, Rafael A. Fighera ${ }^{3}$ e Claudio S. L. Barros ${ }^{3}$
}

\begin{abstract}
Lucena R.B., Fighera R.A. \& Barros C.S.L. 2011. [Peribiliary cysts in an African lion (Panthera leo).] Cistos peribiliares em leão-africano (Panthera leo). Pesquisa Veterinária Brasileira 31(2): 165-168. Departamento de Patologia, Universidade Federal de Santa Maria, 97105-900 Santa Maria, RS, Brazil. E-mail:claudioslbarros@uol.com.br

The gross and histopathological aspects of peribiliary cysts in an adult African lioness (Panthera leo) are described. Grossly, all hepatic lobes contained numerous cysts filled with translucent fluid. Histologically it was observed that the cysts were located in the hepatic hilum and in the portal triads. Periodic acid-Schiff and Alcian blue stains, revealed the cysts to be lined by simple, non-mucinous, cuboidal or columnar epithelium. In immunohistochemistry staining all cells comprising this epithelium were positive for cytokeratin. The lioness died due to complications stemming from a pulmonary carcinoma and no clinical signs related to the hepatic lesion were observed, demonstrating that also in lions the peribiliary cysts occur as non-clinical disease associated lesions.
\end{abstract}

INDEX TERMS: Panthera leo, pathology, hepatology, peribiliary cysts.

RESUMO.- São descritos os achados macroscópicos e histopatológicos de cistos peribiliares em uma fêmea adulta de leão-africano (Panthera leo). Macroscopicamente, todos os lobos hepáticos continham numerosos cistos preenchidos por líquido translúcido. Histologicamente, os cistos estavam localizados no hilo hepático e nos tratos portais. Os cistos eram revestidos internamente por epitélio simples, constituído por células colunares ou cuboidais e não mucinosas, de acordo com avaliação para ácido periódico de Schiff/azul alciano. Na imuno-histiquímica, todas as células desse epitélio foram positivas para citoceratina. O leão morreu de complicações relacionadas a um carcinoma pulmonar e não foram observadas alterações clínicas associadas à lesão hepática, comprovando que cistos peribiliares em leões também ocorrem não associados a sinais clínicos.

TERMOS DE INDEXAÇÃO: Panthera leo, patologia, hepatologia, cistos peribiliares.

\footnotetext{
1 Recebido em 19 de outubro de 2010.

Aceito para publicação em 22 de dezembro de 2010.

2 Programa de Pós-Graduação em Medicina Veterinária, área de concentração em Patologia Veterinária, Centro de Ciências Rurais (CCR), Universidade Federal de Santa Maria (UFSM), Camobi, Santa Maria, RS 97105-900, Brasil. E-mail: lucena.rb@gmail.com

${ }^{3}$ Departamento de Patologia, Centro de Ciências da Saúde, UFSM, Camobi, Santa Maria, RS 97105-900. *Autor para correspondência: claudioslbarros@uol.com.br
}

\section{INTRODUÇÃO}

Cistos peribiliares são cistos de retenção localizados no hilo e nos grandes tratos portais do fígado. Foram descritos primariamente em humanos, como um achado incidental de necropsia (Nakanuma et al. 1984). Posteriormente, a lesão foi associada a doenças hepáticas progressivas, como cirrose, neoplasmas, hipertensão portal idiopática, colangite intra-hepática, infecção sistêmica e doença cística hereditária (Nakanuma 2001, Seguchi et al. 2004). Em Medicina Veterinária, cistos peribiliares são raramente descritos. Há relatos dessa lesão em fígados de suínos sadios, encontradas durante inspeção de abatedouro (Komine et al. 2007, Komine et al. 2008) e associada à letargia, emagrecimento progressivo e morte de um leão (Yu et al. 2007). Este relato tem como objetivo descrever um caso de cistos peribiliares hepáticos em um leão-africano.

\section{MATERIAL E MÉTODOS}

Uma leoa-africana (Panthera leo), adulta, com histórico de dispneia progressiva e perda de peso foi atendido no Hospital Veterinário Universitário da Universidade Federal de Santa Maria (HVU-UFSM), submetido a cirurgia e necropsiado no Laboratório de Patologia Veterinária da UFSM (LPV-UFSM). A causa da morte foi insuficiência respiratória decorrente de um carcinoma pulmonar, padrão bronquíolo-alveolar, difuso, tipo misto (Lucena et al. 2010).

Fragmentos dos cistos hepáticos foram fixados em formol a $10 \%$, embebidos em parafina, seccionados a $3 \mu \mathrm{m}$, corados 
com hematoxilina e eosina (HE) e submetidos a técnicas histoquímicas que incluíram o ácido periódico de Schiff (PAS), azul alciano (AA) e o tricrômico de Masson (TM). Avaliação imunohistoquímica foi realizada pela técnica da estreptavidinabiotina-peroxidase (LSAB Kit Peroxidase Universal, DakoCytomation ${ }^{4}$ ) em diferentes secções do fígado. A imunomarcação foi visualizada com o uso de 3-3'diaminabenzidina (DAB). As secções foram contracoradas com hematoxilina de Harris; utilizaram-se controles positivos e negativos para cada anticorpo. Utilizou-se anticorpo anti-pancitoceratina (policlonal, Dako Cytomation ${ }^{4}$ ), na diluição de 1:2000, e anti-vimentina (clone V9, Dako Cytomation ${ }^{4}$ ), na diluição de 1:100.

\section{RESULTADOS}

Durante a necropsia foram encontradas numerosas massas císticas que variavam de $0,5-15 \mathrm{~cm}$ de diâmetro em todos os lobos hepáticos (Fig.1). Cada cisto era composto por uma cápsula fina e translúcida e continha líquido seroso e claro em seu interior (Fig.2). Histologicamente, os cistos estavam localizados ao longo dos grandes tratos portais intra-hepáticos e no tecido conectivo dos tratos

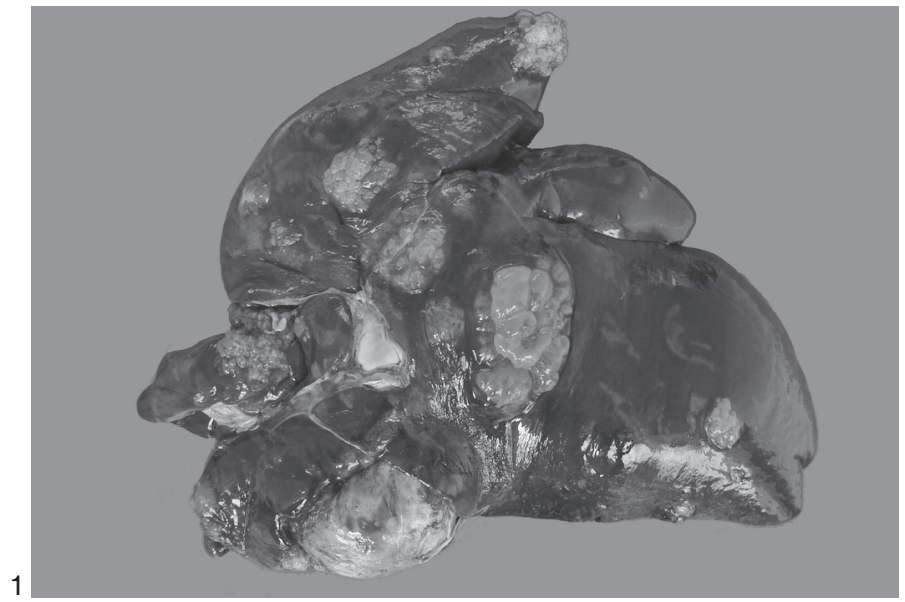

1

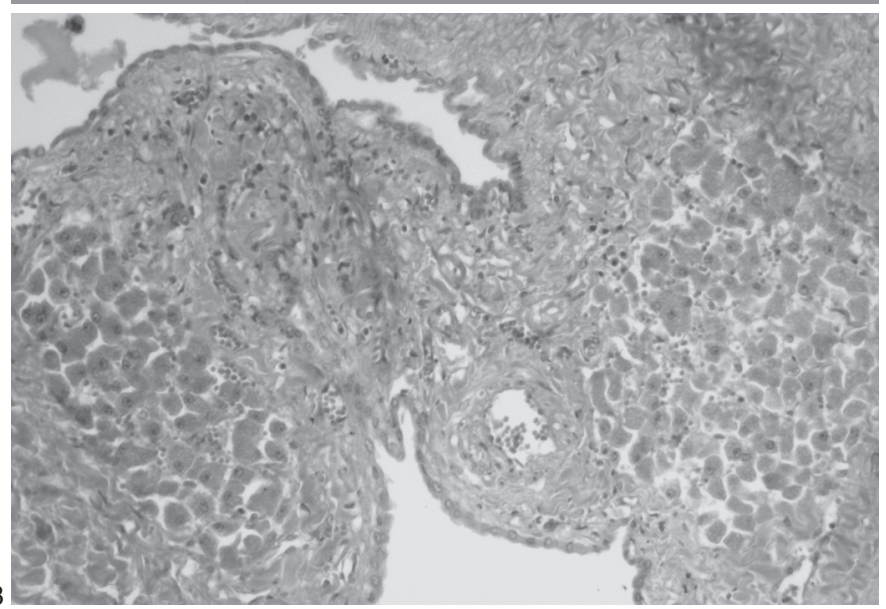

Fig.1. Múltiplos cistos peribiliares são observados em todos os lobos hepáticos de leão africano.

Fig.3. Cistos peribiliares de leão africano, de dimensões variáveis, são vistos envoltos por tecido conjuntivo nos grandes tratos portais. HE, obj.10x. portais (Fig.3). Os cistos eram observados isolados, acentuadamente dilatados, ou em conjunto, como numerosas pequenas dilatações císticas. Todos os cistos eram delineados por células cuboides ou colunares simples (Fig.4), PAS negativas. Circundando as áreas císticas havia abundante proliferação de tecido conjuntivo fibroso, essas áreas marcaram fortemente na avaliação com TM (Fig.5) e foram negativas para AA. Os lóbulos hepáticos adjacentes às áreas císticas estavam comprimidos e tortuosos. Havia moderada proliferação de ductos biliares adjacentes à lesão.

Todas as células cuboidais e colunares que compunham a parede do cisto demonstraram forte e uniforme imunorreatividade citoplasmática para pancitoceratina (Fig.6), o que indica o desenvolvimento das dilatações císticas a partir de estruturas biliares maduras. Na avaliação para vimentina, a expressão foi negativa nas células da parede e positiva no tecido conjuntivo associado ao cisto.

${ }^{4}$ Dako Cytomation, 6392 Via Real, Carpinteria, CA, USA.

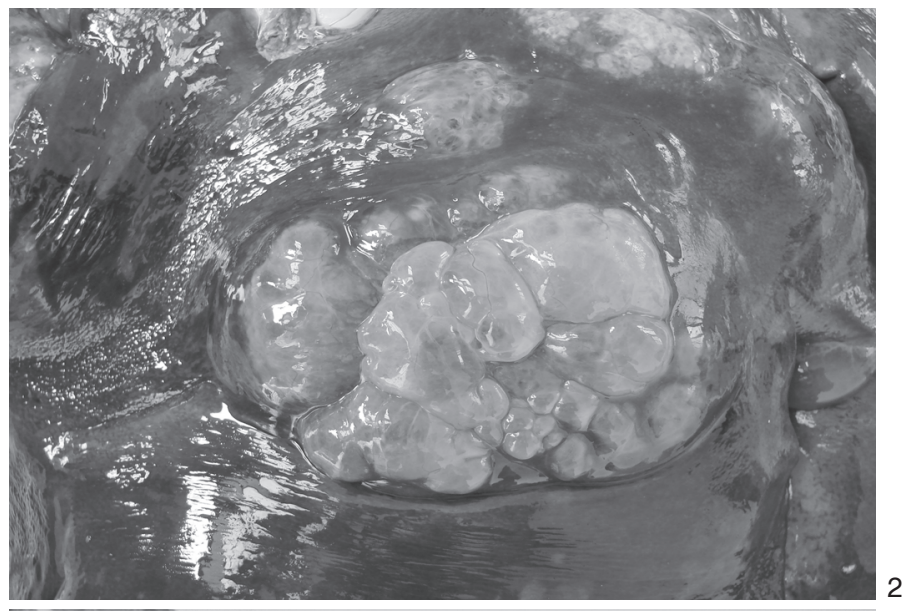

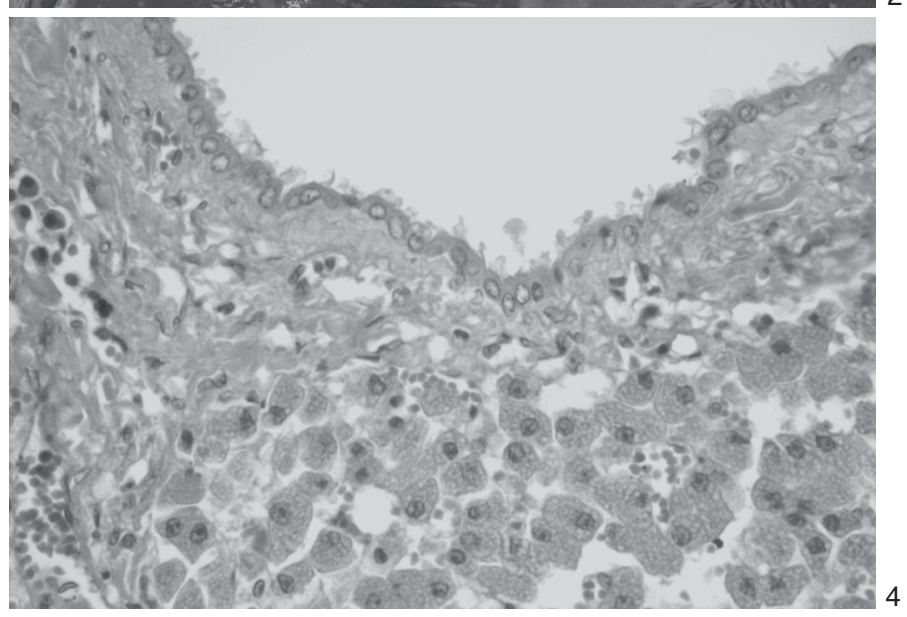

Fig.2. Os cistos peribiliares de leão africano, localizados ao longo dos grandes tratos portais intra-hepáticos, são compostos por cápsula translúcida e possuem tamanhos variados.

Fig.4. Os cistos peribiliares de leão africano são delineados por uma única camada de células cuboidais. HE, obj.20x. 


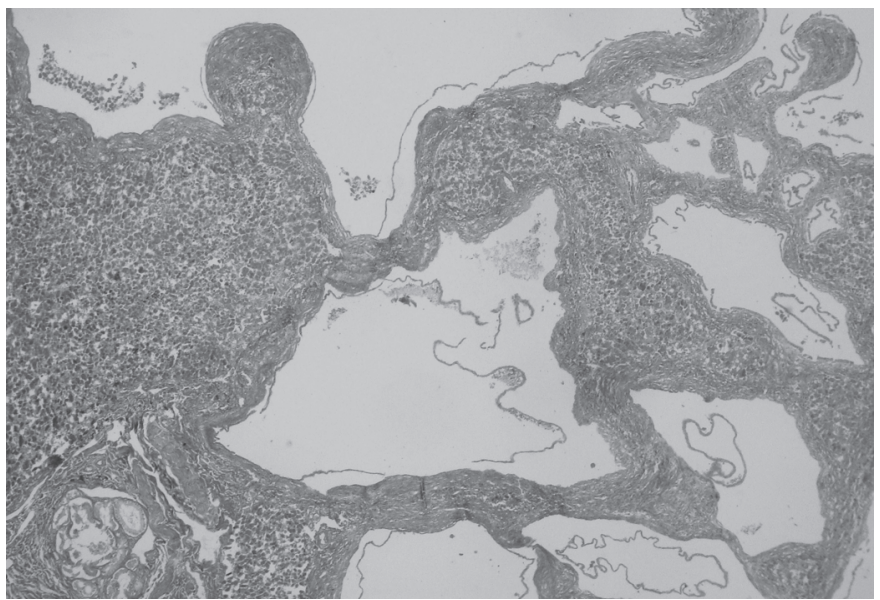

Fig.5. Os cistos peribiliares de leão africano são circundados por abundante quantidade de tecido conjuntivo fibroso que se cora fortemente na avaliação com tricrômico de Masson. TM, obj.20x.

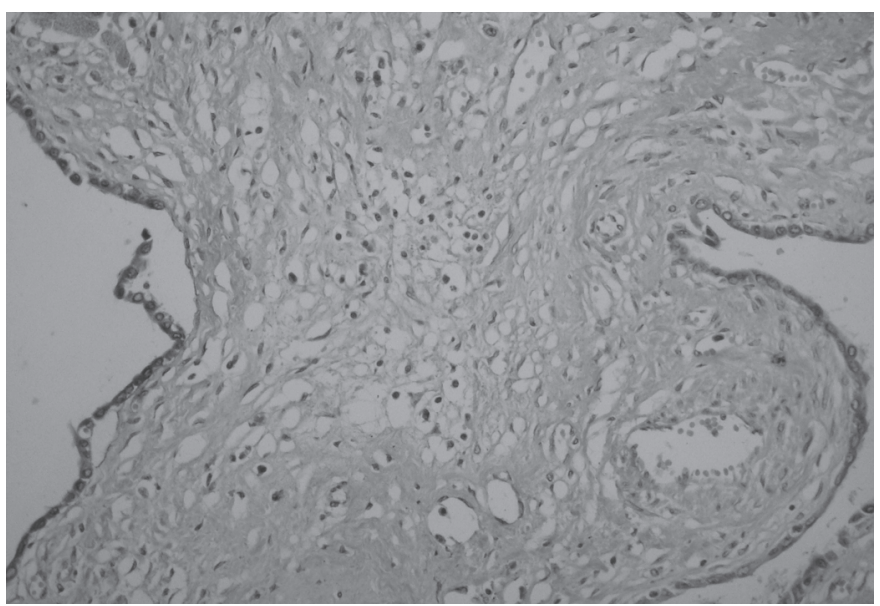

Fig.6. O epitélio cuboidal simples, que compõe a parede dos cistos peribiliares de leão africano, demonstra forte e uniforme imunorreatividade citoplasmática para pancitoceratina. Imuno-histoquímica, método da estreptavidina-biotina-peroxidase, contra-coloração com hematoxilina de Harris, obj.40x.

\section{DISCUSSÃO}

Os achados macroscópicos, histopatológicos, histoquímicos e imuno-histoquímicos confirmam o diagnóstico de cistos peribiliares neste leão. Na literatura, há apenas um relato da referida lesão nesta espécie, associada à doença hepática e responsável pela morte do animal (Yu et al. 2007). No presente caso, a lesão observada no fígado era acentuada e comprometia grande parte do parênquima hepático, no entanto, não foram observados sinais clínicos associados aos cistos peribiliares e a morte ocorreu por outra causa.

Em humanos, em um estudo sistemático do fígado decorrente de 1.000 necropsias, foi constatada a presença de cistos peribiliares em 202 fígados (Terada \& Nakanuma 1990). Na prática clínica essa doença é pouco reconhecida (Nakanuma 2001). Não se conhece a patogenia exata para o surgimento dos cistos peribiliares, mas acredita-se que possam resultar da dilatação cística das glândulas peribiliares que circundam os grandes ductos biliares intra-hepáticos ou, talvez, serem congênitos (Yu et al. 2007).

Cistos peribiliares devem ser diferenciados de outras doenças císticas do fígado, apesar de algumas destas doenças ainda não terem sido descritas em leões. O primeiro diagnóstico diferencial são os cistadenomas biliares (Yu et al. 2007). Estes ocorrem no parênquima hepático, diferente dos cistos peribiliares que se localizam do hilo hepático e nos grandes tratos portais. Nos cistadenomas biliares há pouco conteúdo estromal adjacente, enquanto nos cistos peribiliares, há abundante tecido conjuntivo fibroso. Outra diferença marcante é a intensa produção de mucina pelas células epiteliais que margeiam os cistadenomas biliares e ausência destas células nos cistos peribiliares, como observado no presente caso pela reação negativa com PAS e AA. Além do epitélio dos cistadenomas ser geralmente constituído por mais de uma camada celular, contrário ao epitélio dos cistos peribiliares que é do tipo simples. Em humanos, outro diagnóstico diferencial é o adenofibroma biliar, uma variante morfológica do cistadenoma biliar constituído por microcistos (Salvaggio et al. 2003), diferente dos grandes cistos observados neste relato.

Em suínos, os cistos biliares são importantes diferenciais dos cistos peribiliares (Komine et al. 2008). Os cistos biliares não distorcem o parênquima hepático adjacente, são associados com mínimo tecido fibroso e não é observada proliferação de ductos biliares, diferente da lesão encontrada na leoa.

Outra lesão que deve ser diferenciada dos cistos peribiliares é vista na doença renal policística (DRP) felina, uma doença de caráter hereditário, descrita em gatos da raça Persa, e que se acredita também poder acometer leões (Gerhauser et al., 2009). No entanto, diferente deste caso, na DRP os cistos vistos no fígado não são apenas peribiliares, mas também acometem o parênquima hepático, há mineralização em meio à lesão e sempre o rim é afetado.

Este relato fornece evidências de que cistos peribiliares são também observados como lesões incidentais em leões, semelhante ao descrito em humanos e suínos (Nakanuma et al. 1984, Komine et al. 2007), e que apesar do acentuado acometimento do órgão, tais cistos não necessariamente induzem manifestação clínica. No conhecimento dos autores, esta lesão é raramente descrita em Medicina Veterinária, talvez até por ser confundida com cistadenoma biliares.

\section{REFERÊNCIAS}

Nakanuma Y. 2001. Peribilary cysts: A hitherto poorly recognized disease [Editorial]. J. Gastroenterol. Hepatol. 16(10):1081-1083.

Nakanuma Y., Kurumaya H. \& Ohta G. 1984. Multiple cysts in the hepatic hilum and their pathogenesis. A suggestion of periductal gland origin. Virchows Arch. A Pathol. Anat. Histopathol. 404(4):341-350.

Komine M., Kawasako K., Akihara Y., Shimoyama Y., Okamoto M., Matsuda K., Hirayama K. \& Taniyama H. 2007. Multiple hepatic peribiliary cysts in a young pig. Vet. Pathol. 44(5):707-709.

Komine M., Kawasako K., Okamoto M., Matsuda K., Hirayama K. \& 
Taniyama H. 2008. Histopathological distinction and evolution of biliary and peribiliary cysts in pig liver. J. Comp. Pathol. 139(4):202-207.

Lucena R.B., Fighera R.A., Carregaro A.B., Inkelmann M.A. \& Barros C.S.L. 2010. Carcinoma bronquíolo-alveolar em leão-africano (Panthera leo). Pesq. Vet. Bras. 30(6):479-483.

Seguchi T., Akiyama Y., Itoh H., Tanaka H., Naganuma S., Nagaike K., Uchiyama S. \& Kataoka H. 2004. Multiple hepatic peribiliary cysts with cirrhosis. J. Gastroenterol. 39(4):384-390.
Terada T. \& Nakanuma Y. 1990. Pathological observations of intrahepatic peribiliary glands in 1,000 consecutive autopsy livers. III. Survey of necroinflammation and cystic dilatation. Hepatology 12(5):12291233.

Yu C.H., Kim K.T., Hwang D.N., Yhee J.Y., Moon C.T., Hur T.Y. \& Sur J.H. 2007. Peribilary cysts associated with severe liver disease: A previously unrecognized tumor in a lion (Panthera leo). J. Vet. Diagn. Invest. 19(6):709-712. 